Proyecciones Journal of Mathematics

Vol. 37, No 4, pp. 593-601, December 2018.

Universidad Católica del Norte

Antofagasta - Chile

\title{
Star edge coloring of corona product of path and wheel graph families
}

\author{
Kaliraj $K$. \\ University of Madras, India \\ Sivakami R. \\ Bharathiar University, India \\ and \\ Vernold Vivin J. \\ Anna University Constituent College, India \\ Received: May 2017. Accepted : August 2018
}

\begin{abstract}
A star edge coloring of a graph $G$ is a proper edge coloring without bichromatic paths and cycles of length four. In this paper, we obtain the star edge chromatic number of the corona product of path with cycle, path with wheel, path with helm and path with gear graphs, denoted by $P_{m} \circ C_{n}, P_{m} \circ W_{n}, P_{m} \circ H_{n}, P_{m} \circ G_{n}$ respectively.
\end{abstract}

Keywords : Star edge coloring, corona graph, path, cycle, wheel, helm and gear graph. 


\section{Introduction}

All graphs considered in this paper are finite and simple, i.e. undirected, loopless and without multiple edges. The Maximum degree of a graph $G$ is denoted by $\Delta$.

The corona of two graphs $G_{1}$ and $G_{2}$ is the graph $G=G_{1} \circ G_{2}$ formed from one copy of $G_{1}$ and $\left|V\left(G_{1}\right)\right|$ copies of $G_{2}$ where the $i^{\text {th }}$ vertex of $G_{1}$ is adjacent to every vertex in the $i^{\text {th }}$ copy of $G_{2}$.

For any integer $n \geq 4$, the wheel graph $W_{n}$ is the $n$-vertex graph obtained by joining a vertex $v_{1}$ to each of the $n-1$ vertices $\left\{w_{1}, w_{2}, \ldots w_{n-1}\right\}$ of the cycle graph $C_{n-1}$.

The helm graph $H_{n}$ is the graph obtained from an $(n+1)$-wheel graph by adjoining a pendent edge at each node of the $n$-cycle.

The gear graph $G_{n}$, also known as a bipartite wheel graph, is a $(n+1)$ wheel graph with a graph vertex added between each pair of adjacent graph vertices of the outer cycle.

An edge coloring of graph $G=(V, E)$ is a function $C: E \rightarrow N$, in which any two adjacent edges $e, f \in E$ are assigned different colors. The function $C$ is known as the edge-coloring function. A graph $G$ for which there exists an edge-coloring which requires $k$ colors is called $k$-edge colorable, while such a coloring is called a $k$-edge coloring. The smallest number $k$ of which there exists a $k$-edge-coloring of $G$ is called the chromatic index of a graph $G$ and is denoted by $\chi^{\prime}(G)$.

A star edge coloring of a graph $G$ is a proper edge coloring where at least three distinct colors are used on the edges of every path and cycle of length four, i.e., there is neither bichromatic path nor cycle of length four. The minimum number of colors for which $G$ admits a star edge coloring is called the star edge chromatic index and it is denoted by $\chi_{s t}^{\prime}(G)$.

The star edge coloring was initiated in 2008 by Liu and Deng [8], motivated by the vertex version (see $[1,3,4,6,7,10]$ ). Dvořák, Mohar and Šámal [5] determined upper and lower bounds for complete graphs. L'udmila Bezegová et.al [9] discussed the star edge chromatic number of trees and outerplanar graphs in terms of its maximum degree $\Delta$.

Additional graph theory terminology used in this paper can be found in $[2]$.

In the following section, we discuss the star edge chromatic number of path with cycle, path with wheel, path with helm and path with gear graphs, denoted by $P_{m} \circ C_{n}, P_{m} \circ W_{n}, P_{m} \circ H_{n}, P_{m} \circ G_{n}$ respectively. 


\section{Main Results}

Theorem 2.1. For any positive integer $m$ and $n>4$, then

$$
\chi_{s t}^{\prime}\left(P_{m} \circ C_{n}\right)=\Delta .
$$

Proof. Let $V\left(P_{m}\right)=\left\{u_{i}: 1 \leq i \leq m\right\}$ and

$V\left(C_{n}\right)=\left\{v_{j}: 1 \leq j \leq n\right\}$. Let $E\left(P_{m}\right)=\left\{u_{i} u_{i+1}: 1 \leq i \leq m-1\right\}$ and $E\left(C_{n}\right)=\left\{v_{j} v_{j+1}: 1 \leq j \leq n-1\right\} \cup\left\{v_{n} v_{1}\right\}$. By the definition of corona graph,

$$
\begin{aligned}
V\left(P_{m} \circ C_{n}\right)= & V\left(P_{m}\right) \cup \bigcup_{i=1}^{m}\left\{v_{i j}: 1 \leq j \leq n\right\} \text { and } \\
E\left(P_{m} \circ C_{n}\right)= & E\left(P_{m}\right) \cup \bigcup_{i=1}^{m}\left\{u_{i} v_{i j}: 1 \leq j \leq n\right\} \cup \bigcup_{i=1}^{m}\left\{v_{i j} v_{i j+1}: 1 \leq j \leq n-1\right\} \\
& \cup \bigcup_{i=1}^{m}\left\{v_{i n-1} v_{i 1}\right\} .
\end{aligned}
$$

Let $f$ be a mapping from $E\left(P_{m} \circ C_{n}\right)$ as follows:

Case 1: If $m \geq 3$.

For $1 \leq i \leq m$,

$$
f\left(v_{i j} v_{i j+1}\right)=\left\{\begin{array}{lll}
j+3(\bmod n) & \text { if } & j+3 \not \equiv 0(\bmod n) \\
n(\bmod n) & \text { if } & j+3 \equiv 0(\bmod m+n)
\end{array}\right.
$$

$$
\begin{aligned}
& f\left(u_{3 i-2} u_{3 i-1}\right)=n+1,1 \leq i \leq\left\lceil\frac{m-1}{3}\right\rceil ; f\left(u_{3 i-1} u_{3 i}\right)=n+2,1 \leq \\
& i \leq\left[\frac{m-1}{3}\right] ; f\left(u_{3 i} u_{3 i+1}\right)=n, 1 \leq i \leq\left\lceil\frac{m-1}{3}\right\rceil ; f\left(u_{3 i-2} v_{3 i-2, n}\right)= \\
& n+2,1 \leq i \leq\left\lceil\frac{m-1}{3}\right\rceil ; f\left(u_{3 i-1} v_{3 i-1, n}\right)=n, 1 \leq i \leq\left[\frac{m-1}{3}\right] \\
& f\left(u_{3 i} v_{3 i, n}\right)=n, 1 \leq i \leq\left\lfloor\frac{m}{3}\right\rfloor .
\end{aligned}
$$


Case 2: If $m=2$.

$$
f\left(u_{i} v_{i j}\right)=j, 1 \leq i \leq m, 1 \leq j \leq n ;
$$

$f\left(u_{1} u_{2}\right)=n+1$; and using equation (2.1) and (2.2).

Case 3: If $m=1$, determine $f$ using equation (2.1), (2.2) and (2.3).

It is easy to see that $f$ satisfies no bichromatic 4-path. We assume that $\chi_{s t}^{\prime}\left(P_{m} \circ C_{n}\right) \leq \Delta$. We know that $\chi_{s t}^{\prime}\left(P_{m} \circ C_{n}\right) \geq \chi^{\prime}\left(P_{m} \circ C_{n}\right) \geq \Delta$, since $\chi_{s t}^{\prime}\left(P_{m} \circ C_{n}\right) \geq \Delta$. Therefore $\chi_{s t}^{\prime}\left(P_{m} \circ C_{n}\right)=\Delta$.

Theorem 2.2. For any positive integer $m$ and $n>4$, then

$$
\chi_{s t}^{\prime}\left(P_{m} \circ W_{n}\right)=\Delta .
$$

Proof. Let $V\left(P_{m}\right)=\left\{u_{i}: 1 \leq i \leq m\right\}$ and $V\left(W_{n}\right)=\left\{v_{n}\right\} \cup\left\{v_{j}: 1 \leq j \leq n-1\right\}$. Let $E\left(P_{m}\right)=\left\{u_{i} u_{i+1}: 1 \leq i \leq m-1\right\}$ and $E\left(W_{n}\right)=\left\{v_{n} v_{j}: 1 \leq j \leq n-1\right\} \cup\left\{v_{j} v_{j+1}: 1 \leq j \leq n-2\right\} \cup\left\{v_{n-1} v_{1}\right\}$.

By the definition of corona graph,

$$
\begin{aligned}
V\left(P_{m} \circ W_{n}\right)= & V\left(P_{m}\right) \cup \bigcup_{i=1}^{m}\left\{v_{i j}: 1 \leq j \leq n\right\} \text { and } \\
E\left(P_{m} \circ W_{n}\right)= & E\left(P_{m}\right) \cup \bigcup_{i=1}^{m}\left\{u_{i} v_{i j}: 1 \leq j \leq n\right\} \cup \bigcup_{i=1}^{m}\left\{v_{i n} v_{i j}: 1 \leq j \leq n-1\right\} \\
& \cup \bigcup_{i=1}^{m}\left\{v_{i j} v_{i j+1}: 1 \leq j \leq n-1\right\} \cup \bigcup_{i=1}^{m}\left\{v_{i n-1} v_{i 1}\right\} .
\end{aligned}
$$

Let $f$ be a mapping from $E\left(P_{m} \circ W_{n}\right)$ as follows:

Case 1: If $m \geq 3$.

$$
\left\{\begin{array}{l}
\text { For } 1 \leq i \leq m, \\
f\left(u_{i} v_{i j}\right)=j, 1 \leq j \leq n-1 ; f\left(v_{i n} v_{i j}\right)=j+1,1 \leq j \leq n-2 \\
f\left(v_{i n} v_{i n-1}\right)=1 ; f\left(v_{i j} v_{i j+1}\right)=j+3,1 \leq j \leq n-2
\end{array}\right.
$$




$$
\begin{aligned}
& f\left(v_{i n-1} v_{i 1}\right)=n+2,1 \leq i \leq m ; f\left(u_{3 i-2} u_{3 i-1}\right)=n, 1 \leq i \leq\left\lceil\frac{m-1}{3}\right\rceil ; \\
& f\left(u_{3 i-1} u_{3 i}\right)=n+1,1 \leq i \leq\left[\frac{m-1}{3}\right] ; f\left(u_{3 i} u_{3 i+1}\right)=n+2,1 \leq i \leq \\
& \left\lceil\frac{m-1}{3}\right\rceil ; f\left(u_{3 i-2} v_{3 i-2 n}\right)=n+1,1 \leq i \leq\left\lceil\frac{m}{3}\right\rceil ; f\left(u_{3 i-1} v_{3 i-1 n}\right)= \\
& n+2,1 \leq i \leq\left[\frac{m}{3}\right] ; f\left(u_{3 i} v_{3 i n}\right)=n, 1 \leq i \leq\left\lfloor\frac{m}{3}\right\rfloor .
\end{aligned}
$$

Case 2: If $m=2$.

$$
f\left(u_{1} u_{2}\right)=n+1 ; f\left(v_{i n-1} v_{i 1}\right)=3 \text {; and using equation (2.4). }
$$

Case 3: If $m=1$.

$$
f\left(v_{1 n-1} v_{11}\right)=3 \text {; and using equation (2.4). }
$$

Clearly the above color partitions satisfies no bichromatic 4-path. We assume that $\chi_{s t}^{\prime}\left(P_{m} \circ W_{n}\right) \leq \Delta$. We know that $\chi_{s t}^{\prime}\left(P_{m} \circ W_{n}\right) \geq \chi^{\prime}\left(P_{m} \circ W_{n}\right) \geq$ $\Delta$, since $\chi_{s t}^{\prime}\left(P_{m} \circ W_{n}\right) \geq \Delta$. Therefore $\chi_{s t}^{\prime}\left(P_{m} \circ W_{n}\right)=\Delta$.

Theorem 2.3. For any positive integer $m$ and $n>4$, then

$$
\chi_{s t}^{\prime}\left(P_{m} \circ H_{n}\right)=\Delta .
$$

Proof. Let $V\left(P_{m}\right)=\left\{u_{i}: 1 \leq i \leq m\right\}$ and

$V\left(H_{n}\right)=\left\{v_{n}\right\} \cup\left\{v_{j}: 1 \leq j \leq n-1\right\} \cup\left\{v_{j}^{\prime}: 1 \leq j \leq n-1\right\}$. Let $E\left(P_{m}\right)=$ $\left\{u_{i} u_{i+1}: 1 \leq i \leq m-1\right\}$ and

$E\left(H_{n}\right)=\left\{v_{n} v_{j}: 1 \leq j \leq n-1\right\} \cup\left\{v_{j} v_{j}^{\prime}: 1 \leq j \leq n-1\right\} \cup\left\{v_{j} v_{j+1}: 1 \leq j \leq n-2\right\} \cup$ $\left\{v_{n-1} v_{1}\right\}$. By the definition of corona graph,

$$
\begin{aligned}
V\left(P_{m} \circ H_{n}\right)= & V\left(P_{m}\right) \cup \bigcup_{i=1}^{m}\left\{v_{i n}\right\} \cup \bigcup_{i=1}^{m}\left\{v_{i j}: 1 \leq j \leq n-1\right\} \cup \bigcup_{i=1}^{m}\left\{v_{i j}^{\prime}: 1 \leq j \leq n-1\right\}, \\
E\left(P_{m} \circ H_{n}\right)= & E\left(P_{m}\right) \cup \bigcup_{i=1}^{m}\left\{u_{i} v_{i j}: 1 \leq j \leq n\right\} \cup \bigcup_{i=1}^{m}\left\{u_{i} v_{i j}^{\prime}: 1 \leq j \leq n-1\right\} \\
& \cup \bigcup_{i=1}^{m}\left\{v_{i n} v_{i j}: 1 \leq j \leq n-1\right\} \cup \bigcup_{i=1}^{m}\left\{v_{i j} v_{i j}^{\prime}: 1 \leq j \leq n-1\right\} \\
& \cup \bigcup_{i=1}^{m}\left\{v_{i j} v_{i, j+1}: 1 \leq j \leq n-2\right\} \cup \bigcup_{i=1}^{m}\left\{v_{i, n-1} v_{i 1}\right\} .
\end{aligned}
$$

Let $f$ be a mapping from $E\left(P_{m} \circ H_{n}\right)$ as follows: 
Case 1: If $m \geq 3$.

$$
\left\{\begin{array}{l}
\text { For } 1 \leq i \leq m \\
f\left(u_{i} v_{i j}\right)=j, 1 \leq j \leq n ; f\left(u_{i} v_{j}^{\prime}\right)=n+j, 1 \leq j \leq n-2 \\
f\left(v_{i n} v_{i j}\right)=j+1,1 \leq i \leq n-2 ; f\left(v_{i n} v_{i n-1}\right)=1 \\
f\left(v_{i j} v_{i j+1}\right)=n+j-1,1 \leq j \leq n-2 ; f\left(v_{i n-1} v_{i 1}\right)=2 n-2 \\
f\left(v_{i j} v_{i j}^{\prime}\right)=n+j+1,1 \leq j \leq n-2
\end{array}\right.
$$

$$
\begin{aligned}
& f\left(u_{3 i-2} v_{3 i-2, n-1}^{\prime}\right)=2 n+1,1 \leq i \leq\left\lceil\frac{m}{3}\right\rceil ; f\left(u_{3 i-1} v_{3 i-1, n-1}^{\prime}\right)=2 n- \\
& 1,1 \leq i \leq\left\lceil\frac{m}{3}\right\rceil ; f\left(u_{3 i} v_{3 i, n-1}^{\prime}\right)=2 n, 1 \leq i \leq\left\lfloor\frac{m}{3}\right\rfloor ; f\left(v_{3 i-2, n-1} v_{3 i-2, n-1}^{\prime}\right)= \\
& 2 n, 1 \leq i \leq\left\lceil\frac{m}{3}\right] ; f\left(v_{3 i-1, n-1} v_{3 i-1, n-1}^{\prime}\right)=2 n+1,1 \leq i \leq\left\lceil\frac{m}{3}\right] \\
& f\left(v_{3 i, n-1} v_{3 i, n-1}^{\prime}\right)=2 n-1,1 \leq i \leq\left\lfloor\frac{m}{3}\right\rfloor ; f\left(u_{3 i-2} u_{3 i-1}\right)=2 n, 1 \leq \\
& i \leq\left\lceil\frac{m-1}{3}\right\rceil ; f\left(u_{3 i-1} u_{3 i}\right)=2 n+1,1 \leq i \leq\left\lfloor\frac{m-1}{3}\right\rfloor ; f\left(u_{3 i} u_{3 i+1}\right)= \\
& 2 n-1,1 \leq i \leq\left\lceil\frac{m-1}{3}\right\rceil .
\end{aligned}
$$

Case 2: If $m=2$.

$$
\begin{aligned}
& f\left(u_{1} u_{2}\right)=2 n ; f\left(v_{1, n-1} v_{1, n-1}^{\prime}\right)=2 n ; f\left(u_{1} v_{1, n-1}^{\prime}\right) \\
& =2 n+1 ; f\left(u_{2} v_{2, n-1}^{\prime}\right)=2 n-1 \text { and using equation }(2.5) .
\end{aligned}
$$

Case 3: If $m=1$.

$$
f\left(v_{1, n-1} v_{1, n-1}^{\prime}\right)=2 n ; f\left(u_{1} v_{1, n-1}^{\prime}\right)=2 n+1 \text { and using equation }
$$

Clearly the above color partitions satisfies no bichromatic 4-path. We assume that $\chi_{s t}^{\prime}\left(P_{m} \circ H_{n}\right) \leq \Delta$. We know that $\chi_{s t}^{\prime}\left(P_{m} \circ H_{n}\right) \geq \chi^{\prime}\left(P_{m} \circ H_{n}\right) \geq \Delta$, since $\chi_{s t}^{\prime}\left(P_{m} \circ H_{n}\right) \geq \Delta$. Therefore $\chi_{s t}^{\prime}\left(P_{m} \circ H_{n}\right)=\Delta$.

Theorem 2.4. For any positive integer $m$ and $n \geq 5$, then

$$
\chi_{s t}^{\prime}\left(P_{m} \circ G_{n}\right)=\Delta .
$$


Proof. Let $V\left(P_{m}\right)=\left\{u_{i}: 1 \leq i \leq m\right\}$ and

$V\left(G_{n}\right)=\left\{v_{n}\right\} \cup\left\{v_{j}: 1 \leq j \leq n-1\right\} \cup\left\{v_{j}^{\prime}: 1 \leq j \leq n-1\right\}$. Let $E\left(P_{m}\right)=$ $\left\{u_{i} u_{i+1}: 1 \leq i \leq m-1\right\}$ and

$E\left(G_{n}\right)=\left\{v_{n} v_{j}: 1 \leq j \leq n-1\right\} \cup\left\{v_{j} v_{j}^{\prime}: 1 \leq j \leq n-1\right\} \cup\left\{v_{j}^{\prime} v_{j+1}: 1 \leq j \leq n-1\right\} \cup$

$\left\{v_{n-1}^{\prime} v_{1}\right\}$. By the definition of corona graph,

$$
\begin{aligned}
V\left(P_{m} \circ G_{n}\right)= & V\left(P_{m}\right) \cup \bigcup_{i=1}^{m}\left\{v_{i n}\right\} \cup \bigcup_{i=1}^{m}\left\{v_{i j}: 1 \leq j \leq n-1\right\} \cup \bigcup_{i=1}^{m}\left\{v_{i j}^{\prime}: 1 \leq j \leq n-1\right\}, \\
E\left(P_{m} \circ G_{n}\right)= & E\left(P_{m}\right) \cup \bigcup_{i=1}^{m}\left\{u_{i} v_{i j}: 1 \leq j \leq n\right\} \cup \bigcup_{i=1}^{m}\left\{u_{i} v_{i j}^{\prime}: 1 \leq j \leq n-1\right\} \\
& \cup \bigcup_{i=1}^{m}\left\{v_{i n} v_{i j}: 1 \leq j \leq n-1\right\} \cup \bigcup_{i=1}^{m}\left\{v_{i j} v_{i j}^{\prime}: 1 \leq j \leq n-1\right\} \\
& \cup \bigcup_{i=1}^{m}\left\{v_{i j}^{\prime} v_{i, j+1}: 1 \leq j \leq n-2\right\} \cup \bigcup_{i=1}^{m}\left\{v_{i, n-1}^{\prime} v_{i 1}\right\} .
\end{aligned}
$$

Let $f$ be a mapping from $E\left(P_{m} \circ G_{n}\right)$ as follows:

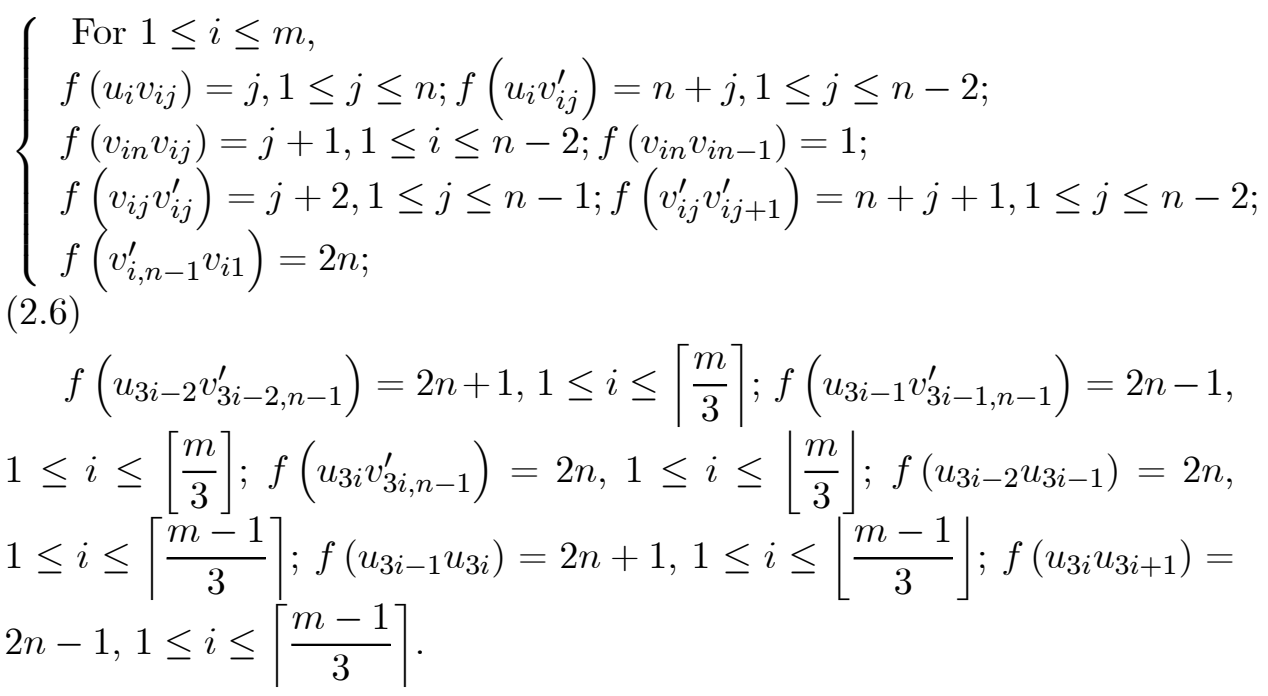

Clearly the above color partitions satisfies no bichromatic 4-path. We assume that $\chi_{s t}^{\prime}\left(P_{m} \circ G_{n}\right) \leq \Delta$. We know that $\chi_{s t}^{\prime}\left(P_{m} \circ G_{n}\right) \geq \chi^{\prime}\left(P_{m} \circ G_{n}\right) \geq$ $\Delta$, since $\chi_{s t}^{\prime}\left(P_{m} \circ G_{n}\right) \geq \Delta$. Therefore $\chi_{s t}^{\prime}\left(P_{m} \circ G_{n}\right)=\Delta$. 


\section{Acknowledgements}

The authors are grateful to the referee for his valuable suggestions, comments and corrections that have resulted in the improvement of this paper.

\section{References}

[1] Albertson, M. O., Chappell, G. G., Kiersted, H. A., Künden, A., and Ramamurthi, R. Coloring with no 2-colored $P_{4}$ 's. Electron. J. Combin. 1 (2004), \#R26.

[2] Bondy,J. A., Murty, U.S.R., Graph Theory with Applications, London, Macmillan, (1976).

[3] Bu, Y., Cranston, N. W., Montassier, M., Raspaud, A., and Wang, W. Star-coloring of sparse graphs. J. Graph Theory 62, pp. 201-219, (2009).

[4] Chen, M., Raspaud, A., and Wang, W. 6-star-coloring of subcubic graphs. J. Graph Theory 72, 2, pp. 128-145, (2013).

[5] Dvořák, Z., Mohar, B., and Šámal, R. Star chromatic index. J. Graph Theory 72, pp. 313-326, (2013).

[6] Grünbaum, B. Acyclic coloring of planar graphs. Israel J. Math. 14, pp. 390-412, (1973).

[7] Kierstead, H. A., Kündgen, A., and Timmons, C. Star coloring bipartite planar graphs. J. Graph Theory 60, pp. 1-10, (2009).

[8] Liu, X.S., and Deng, K. An upper bound on the star chromatic index of graphs with $\delta \geq 7$. J. Lanzhou Univ. (Nat. Sci.) 44, pp. 94-95, (2008).

[9] L’udmila Bezegová, Borut Lužar, Martina Mockovciaková, Roman Soták, Riste Śkrekovski, Star Edge Coloring of Some Classes of Graphs, Journal of Graph Theory, Article first published online: 18 FEB 2015 - DOI: 10.1002/jgt.21862.

[10] Nešetřil, J. and De Mendez, P. O. Colorings and homomorphisms of minor closed classes. Algorithms Combin. 25, pp. 651-664, (2003). 


\section{Kaliraj K.}

Ramanujan Institute for Advanced Study in Mathematics, University of Madras, Chepauk, Chennai-600 005, Tamil Nadu, India

e-mail : sk.kaliraj@gmail.com

\section{Sivakami R.}

Part-Time Research Scholar (Category-B)

Research \& Development Centre

Bharathiar University

Coimbatore 641046

Tamil Nadu,

India

e-mail : sivakawin@gmail.com ;

and

\section{Vernold Vivin J.}

Department of Mathematics, University College of Engineering Nagercoil, (Anna University Constituent College), Konam, Nagercoil-629 004,

Tamil Nadu, India

e-mail : vernoldvivin@yahoo.in 